\title{
ERRATUM: \\ FIRST PASSAGE PERCOLATION ON RANDOM GRAPHS WITH FINITE MEAN DEGREES \\ [Ann. Appl. Probab. 20(5) (2010) 1907-1965]
}

\author{
By SHANKAR BHAMIDI ${ }^{1}$, REMCO VAN DER HOFSTAD ${ }^{2}$ AND \\ GERARD HOOGHIEMSTRA \\ University of North Carolina, Eindhoven University of Technology and \\ Delft University of Technology
}

\begin{abstract}
In this erratum, we correct a mistake in the above paper, where we were using an exchangeability result that is obviously false.
\end{abstract}

1. Description of the error and where it is used. In [4], we considered first passage percolation for configuration model random graphs with i.i.d. degrees, where each edge in the constructed graph is assigned an exponential mean one weight, independently across edges. The aim was to understand both the weight and the number of edges (hopcount) on the optimal path between two uniformly chosen vertices, which we will occasionally call sources, thinking of fluid percolating through the network at rate one, started at the two source vertices. When these two explorations meet (collide), they generate the shortest path between these two sources. In the sequel, these exploration processes will sometimes be referred to as smallest-weight trees from the two source vertices. Using the fact that in the construction the edges have exponentially distributed weights resulted in an explicit description of this exploration of the graph in [4]. A rigorous analysis of this process gave rise to the results in the paper.

One technical result in the analysis was [4], Lemma 6.1, where we claimed that the sequence of random variables $\left(B_{R_{m}}\right)_{m \geq 1}$ is exchangeable, which is false. Here, $\left(B_{R_{m}}\right)_{m \geq 1}$ are the forward degrees in the exploration of a connected component of a uniformly chosen vertex $U \in[n]=\{1, \ldots, n\}$ in the configuration model with i.i.d. degrees, ignoring $U$ itself. Indeed, conditionally on the degrees $\left(D_{i}\right)_{i \in[n]}$, the sequence $\left(B_{R_{m}}\right)_{m \geq 1}$ is a size-biased reordering of the random variables $\left(D_{i}-\right.$ $1)_{i \in[n] \backslash\{U\}}$. Thus, in particular, the expected values of $\left(B_{R_{m}}\right)_{m \geq 1}$ are decreasing

\footnotetext{
Received December 2016.

${ }^{1}$ Supported in part by NSF-DMS 2569 Grants 1105581, 1310002, 160683, 161307, SES Grant 1357622 and AROW911NF-17-1-0010.

${ }^{2}$ Supported by NWO VICI Grant 639.033 .806 and NWO Gravitation Grant Networks 024.002.003.

MSC2010 subject classifications. Primary 60C05; secondary 05C80, 90B15.

Key words and phrases. Flows, random graph, first passage percolation, hopcount, central limit theorem, configuration model.
} 
in $m$, so they cannot be exchangeable. This lemma is crucially used in [4], Proof of Proposition 4.6(a). In this erratum, we correct this proof.

Since the appearance of [4], new results for first passage percolation (FPP) on the configuration model (CM) have appeared in rather general setting. We refer to [3], where we have investigated FPP on the CM for general, but continuous edgeweight distributions in the case where the degrees have a finite second moment (with an extra logarithm so that the branching-process approximation satisfies the $X \log X$ condition in the Kesten-Stigum theorem) and are i.i.d. Since the exponential weight distribution, which we assume in [4], is continuous, the proofs given in [3] imply those in [4], in the case where the degrees have a finite $X^{2} \log X$ moment. In [4], we have assumed that the degrees either obey a power law with exponents $\tau \in(2,3)$, or a distribution whose tail distribution can be bounded from above by a power law with exponent $\tau>3$. In the latter case, [4], Lemma 6.1, is not used, and thus there is nothing to correct.

The aim of this paper is to correct the argument in [4] that uses [4], Lemma 6.1. This argument arises in the analysis of the regime $\tau \in(2,3)$. In this regime, no general results are known for the number of edges in the smallest-weight path between two uniform vertices, even though more general results have been proved for the weight-distance (see $[1,2]$ ). The proofs of the results in these papers do not rely on [4], Lemma 6.1. Thus, for $\tau \in(2,3)$, the asserted central limit result [4], Theorem 3.2, has to be reproved. The only place where [4], Lemma 6.1, was used in the proof of [4], Theorem 3.2, was in the proof of [4], Proposition 4.6(a). It is hence sufficient to reprove [4], Proposition 4.6(a), for the case where $\tau \in(2,3)$. In fact, we will prove a slightly weaker version of [4], Proposition 4.6(a), and will explain how this version suffices for the proof of [4], Theorem 3.2.

2. Correction of [4], Proposition 4.6(a). We start by recalling some of the definitions in [4]. The distribution function $F$ of the i.i.d. degrees $D_{1}, D_{2}, \ldots$ is assumed to satisfy

$$
c_{1} x^{-(\tau-1)} \leq 1-F(x) \leq c_{2} x^{-(\tau-1)}, \quad x \geq 1,
$$

where $\tau \in(2,3)$, and for some constants $0<c_{1} \leq c_{2}<\infty$ (compare [4], (3.8)). Furthermore, we recall that $a_{n}$ is the power of $n$ that indicates the maximal size of each of the trees grown from the sources $U_{i}, i=1,2$, at the moment when the smallest-weight path is being formed (i.e., the collision time), equals

$$
a_{n}=n^{(\tau-2) /(\tau-1)} \quad \text { for } \tau \in(2,3) .
$$

We also recall from [4] the random variables involved in [4], Proposition 4.6 and Lemma 6.1. Let $S_{0}=1, S_{1}=D_{1}$, and, for $j \geq 2$,

$$
S_{j}=D_{1}+\sum_{i=2}^{j}\left(B_{i}-1\right),
$$


where, in case the chosen half-edge is real, that is, not artificial, and the paired half-edge is not one of the allowed half-edges, $B_{i}$ equals the forward degree of the vertex incident to the $i$ th paired half-edge, whereas $B_{i}=0$ otherwise. Finally, we recall that, conditionally on $\vec{B}=\left(D_{1}, B_{2}, B_{3}, \ldots, B_{m}\right)$ and for $2 \leq j \leq m$,

$$
G_{m}=\sum_{i=1}^{m} I_{i} \quad \text { where } \quad \mathbb{P}\left(I_{1}=1 \mid \vec{B}\right)=1, \mathbb{P}\left(I_{j}=1 \mid \vec{B}\right)=B_{j} / S_{j}
$$

We now turn to the proof of [4], Proposition 4.6(a). Let us start by recalling the content of [4], Proposition 4.6(a).

Reference [4], Proposition 4.6(a), states that, for $\tau \in(2,3)$ and with $m \leq \bar{m}_{n}$, for any $\bar{m}_{n}$ such that $\log \left(\bar{m}_{n} / a_{n}\right)=o(\sqrt{\log n})$,

$$
\frac{G_{m}-\log m}{\sqrt{\log m}} \stackrel{d}{\longrightarrow} Z \quad \text { where } Z \sim \mathcal{N}(0,1)
$$

i.e., $Z$ has a standard normal distribution. Here, we have used that the parameter $\beta$ in [4], Proposition 4.6(a), takes the value 1 for $\tau \in(2,3)$ (see [4], Proposition 4.3).

We will not prove precisely this version of [4], Proposition 4.6(a), but instead prove that there exists $\bar{m}_{n} \gg a_{n}$ such that for all $m \leq \bar{m}_{n}$ the CLT in (2.5) holds. That is enough, since [4], Proposition 4.6(a), is used for $\bar{m}_{n}=\mathrm{C}_{n}$ (recall [4], (4.30)), the collision time. By [4], Lemma 7.2, $\mathrm{C}_{n}=o \mathbb{P}\left(\bar{m}_{n}\right)$ for any $\bar{m}_{n} \gg a_{n}$, so this slightly weaker version indeed suffices to prove [4], Theorem 3.2. Now we adapt the proof.

Proof of [4], Proposition 4.6(A). We start with the initial steps of the proof, which can be performed as before in [4].

Step 1: Coupling to an $n$-dependent i.i.d. sequence. Take $\underline{m}_{n}=o\left(a_{n}\right)$ such that $\log \left(a_{n} / \underline{m}_{n}\right)=o(\sqrt{\log n})$ and let $L_{n}=\sum_{i \in[n]} D_{i}$. Then, as in the original proof of the proposition where [4], Lemma 6.1, was not used, the sequence $\left(B_{i}\right)_{i=1}^{\underline{m}_{n}}$ can be coupled to an i.i.d. sequence $\left(\widetilde{B}_{i}\right)_{i=1}^{\underline{m}}$. Note that the distribution of $\widetilde{B}_{i}$ depends on $n$, as it is given by

$$
\mathbb{P}_{n}\left(\widetilde{B}_{i}=k\right)=\frac{k+1}{L_{n}} \mathbb{1}_{\left\{D_{i}=k+1\right\}},
$$

where $\mathbb{P}_{n}$ denotes the conditional probability of the $C M$, given the degrees $\left(D_{i}\right)_{i \in[n]}$. Let us explain this in more detail. See also [3], Section 2, where this coupling is worked out in full detail. The law in (2.6) corresponds to the forward degree of a half-edge chosen uniformly at random from the collection of $L_{n}=\sum_{i \in[n]} D_{i}$ half-edges. We grow the smallest-weight graph (SWG) and the $\mathrm{CM}$ at the same time. We investigate what happens when we pair $\underline{m}_{n}$ half-edges. The distinction between the law of $\left(B_{i}\right)_{i=1}^{\underline{m}_{n}}$ and the i.i.d. sequence $\left(\widetilde{B}_{i}\right)_{i=1}^{\underline{m}_{n}}$ is due to the fact that the number of half-edges decreases (which we refer to as half-edge 
reuse), and the fact that $B_{i} \neq \widetilde{B}_{i}$ when the vertex incident to the half-edge has previously been chosen (which we refer to as vertex reuse). We claim that w.h.p. there is no half-edge nor vertex reuse in the $\underline{m}_{n}$ first pairings. Indeed, the probability of a half-edge or vertex reuse in the $\underline{m}_{n}$ first pairings is bounded above by

$$
\underline{m}_{n}^{2} \sum_{i \in[n]} \frac{D_{i}^{2}}{L_{n}^{2}}
$$

since at most $\underline{m}_{n}^{2}$ half-edges are involved. To see (2.7), fix a half-edge $x_{i}$ incident to vertex $i$. The probability that it is chosen at any time equals $1 / L_{n}$, the probability that it or any other half-edge incident to the same vertex is chosen again (thus creating a half-edge or vertex reus) is $D_{i} / L_{n}$. The union bound then completes the proof of (2.7).

When $\left(D_{i}\right)_{i \in[n]}$ are i.i.d. with distribution function $F$ as in (2.1),

$$
n^{-2 /(\tau-1)} \sum_{i \in[n]} D_{i}^{2} \stackrel{d}{\longrightarrow} \mathcal{S}_{\tau-2},
$$

where $\mathcal{S}_{\tau-2}$ has an $\alpha$-stable distribution with $\alpha=\tau-2$ (see [5], IX.8). Thus,

$$
\left(n^{-(\tau-2) /(\tau-1)} \underline{m}_{n}\right)^{2} n^{-2 /(\tau-1)} \sum_{i \in[n]} D_{i}^{2} \stackrel{\mathbb{P}}{\longrightarrow} 0 .
$$

Because of this and since $L_{n} / n \stackrel{\text { a.s. }}{\longrightarrow} \mathbb{E}[D]$, the object in (2.7) converges to zero in probability as $n \rightarrow \infty$. This proves the claim and completes the first step.

Step 2: Consequences of the coupling. As a consequence of the above coupling, we note that $S_{\underline{m}_{n}}$ can be coupled to $\widetilde{S}_{\underline{m}_{n}}$, where

$$
\widetilde{S}_{\underline{m}_{n}} \geq \max _{i \leq \underline{m}_{n}} \widetilde{B}_{i} \equiv \widetilde{M}_{\underline{m}_{n}}^{(n)},
$$

where we have used that $\widetilde{B}_{i} \geq 1$ so that the summands in (2.3) are non-negative. In turn, we can couple $\widetilde{M}_{\underline{m}_{n}}^{(n)}$ to $M_{\underline{m}_{n}}=\max _{i \leq \underline{m}_{n}} B_{i}$. In the following lemma, we investigate the asymptotics of these maxima.

LEMMA 2.1 (Lower bound on $M_{\underline{m}_{n}}$ and $\widetilde{M}_{\underline{m}_{n}}^{(n)}$ ). Take $\varepsilon_{n}=o(1)$ and $\underline{m}_{n}=$ $\varepsilon_{n}^{\kappa} a_{n}$ with $\kappa \in(0, \tau-2)$. Then the following two statements hold w.h.p.:

$$
M_{\underline{m}_{n}} \geq \varepsilon_{n} n^{1 /(\tau-1)} \quad \text { and } \quad \widetilde{M}_{\underline{m}_{n}}^{(n)} \geq \varepsilon_{n} n^{1 /(\tau-1)} .
$$

PROOF. We only prove the second statement, the first statement then follows from the coupling. Observe that

$$
\begin{aligned}
\mathbb{P}_{n}\left(\widetilde{M}_{\underline{m}_{n}}^{(n)}<\varepsilon_{n} n^{1 /(\tau-1)}\right) & =\mathbb{P}_{n}\left(\widetilde{B}_{i} \leq \varepsilon_{n} n^{1 /(\tau-1)}-1\right)^{\underline{m}_{n}} \\
& \equiv F_{n}^{\star}\left(\varepsilon_{n} n^{1 /(\tau-1)}-1\right)^{\underline{m}_{n}},
\end{aligned}
$$


where

$$
F_{n}^{\star}(x)=\sum_{i \in[n]} \frac{D_{i}}{L_{n}} \mathbb{1}_{\left\{D_{i} \leq x+1\right\}}
$$

We note that

$$
1-F_{n}^{\star}(x)=\sum_{i \in[n]} \frac{D_{i}}{L_{n}} \mathbb{1}_{\left\{D_{i}>x+1\right\}}>\frac{x+1}{L_{n} / n}\left[1-F_{n}\right](x+1),
$$

where $F_{n}$ is the empirical distribution function of the degrees given by

$$
F_{n}(x)=\frac{1}{n} \sum_{i \in[n]} \mathbb{1}_{\left\{D_{i} \leq x\right\}}
$$

We conclude that

$$
\mathbb{P}_{n}\left(\widetilde{M}_{\underline{m}_{n}}^{(n)}<\varepsilon_{n} n^{1 /(\tau-1)}\right) \leq\left(1-\frac{\varepsilon_{n} n^{1 /(\tau-1)}}{L_{n} / n}\left[1-F_{n}\right]\left(\varepsilon_{n} n^{1 /(\tau-1)}\right)\right)^{\underline{m}_{n}}
$$

First, $L_{n} / n \stackrel{\text { a.s. }}{\longrightarrow} \mathbb{E}[D]$, so that w.h.p. $L_{n} / n \leq 2 \mathbb{E}[D]$.

Next, note that $n\left[1-F_{n}\right]\left(\varepsilon_{n} n^{1 /(\tau-1)}\right)$ has a binomial distribution with $n$ trials and success probability

$$
[1-F]\left(\varepsilon_{n} n^{1 /(\tau-1)}\right) \geq c_{1}\left(\varepsilon_{n} n^{1 /(\tau-1)}\right)^{-(\tau-1)}=c_{1} \varepsilon_{n}^{-(\tau-1)} n^{-1},
$$

where we have used (2.1). Therefore,

$$
\mathbb{E}\left[n\left[1-F_{n}\right]\left(\varepsilon_{n} n^{1 /(\tau-1)}\right)\right] \geq c_{1} \varepsilon_{n}^{-(\tau-1)} \rightarrow \infty,
$$

so that $n\left[1-F_{n}\right]\left(\varepsilon_{n} n^{1 /(\tau-1)}\right) \geq c_{1} \varepsilon_{n}^{-(\tau-1)} / 2$, w.h.p. We conclude that, w.h.p., and for some $\tilde{c}_{1}>0$,

$$
1-\frac{\varepsilon_{n} n^{1 /(\tau-1)}}{L_{n} / n}\left[1-F_{n}\right]\left(\varepsilon_{n} n^{1 /(\tau-1)}\right) \leq 1-\tilde{c}_{1} \varepsilon_{n}^{-(\tau-2)} n^{-(\tau-2) /(\tau-1)} .
$$

Therefore, again w.h.p. and using that $\underline{m}_{n}=\varepsilon_{n}^{\kappa} a_{n}$ and $a_{n}=n^{(\tau-2) /(\tau-1)}$,

$$
\begin{aligned}
\mathbb{P}_{n}\left(\widetilde{M}_{\underline{m}_{n}}^{(n)}<\varepsilon_{n} n^{1 /(\tau-1)}\right) & \leq\left(1-\tilde{c}_{1} \varepsilon_{n}^{-(\tau-2)} n^{-(\tau-2) /(\tau-1)}\right)^{\underline{m}_{n}} \\
& \leq \mathrm{e}^{-\tilde{c}_{1} \varepsilon_{n}^{\kappa-(\tau-2)}}=o(1),
\end{aligned}
$$

whenever $\kappa \in(0, \tau-2)$.

Step 3: Reduction of the proof to a first moment computation. By the above (and recall, e.g., [4], (6.5)), the proof is completed when we show that, with $\mathcal{E}_{n}=$ $\left\{M_{\underline{m}_{n}} \geq \varepsilon_{n} n^{1 /(\tau-1)}\right\}$,

$$
\mathbb{1}_{\mathcal{E}_{n}} \frac{\sum_{i=\underline{m}_{n}+1}^{\bar{m}_{n}} I_{i}}{\sqrt{\log n}} \stackrel{\mathbb{P}}{\longrightarrow} 0
$$


for appropriate $\underline{m}_{n}, \bar{m}_{n}$ satisfying $\log \left(a_{n} / \underline{m}_{n}\right)=o(\sqrt{\log n})$ and $\log \left(\bar{m}_{n} / a_{n}\right)=$ $o(\sqrt{\log n})$. Indeed, for $j \leq \underline{m}_{n}$, we can couple the indicators $I_{j}$ to an independent sequence $\left(\hat{I}_{j}\right)_{j \leq \underline{m}_{n}}$, where, conditionally on the degrees $\left(D_{i}\right)_{i \in[n]}$, the random variables $\left(\hat{I}_{j}\right)_{j \leq \underline{m}_{n}}$ are independent, with

$$
\mathbb{P}_{n}\left(\hat{I}_{j}=1\right)=\frac{\widetilde{B}_{i}}{\sum_{i \leq j} \widetilde{B}_{j}},
$$

and $\left(I_{j}\right)_{j \leq \underline{m}_{n}}$ are also unconditionally independent with

$$
\mathbb{E}\left[\mathbb{P}_{n}\left(\hat{I}_{j}=1 \mid \vec{B}\right)\right]=\mathbb{E}\left[\frac{\widetilde{B}_{i}}{\sum_{i \leq j} \widetilde{B}_{j}}\right]=\frac{1}{j}, \quad 2 \leq j \leq m .
$$

The unconditional independence of the indicators $\left(\hat{I}_{j}\right)_{j \leq \underline{m}_{n}}$ is proved in [4], (5.4). Further, with

$$
\hat{G}_{m}=\sum_{j=1}^{m} \hat{I}_{j}
$$

we obtain that for any $\kappa_{n} \rightarrow \infty$,

$$
\mathbb{P}_{n}\left(\left|G_{\underline{m}_{n}}-\hat{G}_{\underline{m}_{n}}\right| \geq \kappa_{n}\right)=o_{\mathbb{P}}(1) .
$$

Since $\hat{G}_{\underline{m}_{n}}$ is a sum of independent indicators, the proof as in [4], Proof of Proposition 4.3(a), which relies on the Lindeberg-Lévy-Feller CLT, can be copied verbatim to show that $\left(G_{\underline{m}_{n}}-\log \underline{m}_{n}\right) / \sqrt{\log \underline{m}_{n}} \stackrel{d}{\longrightarrow} Z$. This proves [4] subject to (2.21), Proposition 4.6(a).

Step 4: Completion of the proof. Our strategy will be to calculate an upper bound for the expected value of the random quantity in (2.21) and show that this upper bound converges to 0 . We will take $\underline{m}_{n}=\varepsilon_{n}^{\kappa} a_{n}$ and $\bar{m}_{n}=\varepsilon_{n}^{-\kappa} a_{n}$, where $\kappa \in(0, \tau-$ 2) and $\varepsilon_{n} \gg(\log n)^{-1 /(2+2 \kappa)}$. We will always work under the conditional measure $\mathbb{P}_{n}$ given the degrees.

Denote the $\sigma$-algebra given $D_{1}, B_{2}, \ldots, B_{i}$ by $\mathcal{F}_{i}$. Then, for $i \geq 2$,

$$
\mathbb{E}_{n}\left[I_{i} \mid \mathcal{F}_{i}\right]=\frac{B_{i}}{S_{i}}
$$

Therefore,

$$
\begin{aligned}
\mathbb{E}_{n}\left[\mathbb{1}_{\left.\mathcal{E}_{n} \sum_{i=\underline{m}_{n}+1}^{\bar{m}_{n}} I_{i}\right]}=\sum_{i=\underline{m}_{n}+1}^{\bar{m}_{n}} \mathbb{E}_{n}\left[\mathbb{1}_{\mathcal{E}_{n}} I_{i}\right]\right. \\
=\sum_{i=\underline{m}_{n}+1}^{\bar{m}_{n}} \mathbb{E}_{n}\left[\mathbb{1}_{\mathcal{E}_{n}} \mathbb{E}_{n}\left[I_{i} \mid \mathcal{F}_{i}\right]\right] \\
=\sum_{i=\underline{m}_{n}+1}^{\bar{m}_{n}} \mathbb{E}_{n}\left[\mathbb{1}_{\mathcal{E}_{n}} \frac{B_{i}}{S_{i}}\right],
\end{aligned}
$$

where we have also used that $\mathcal{E}_{n}$ is $\mathcal{F}_{i}$-measurable when $i>\underline{m}_{n}$. 
We note that, by Lemma 2.1 and w.h.p.,

$$
S_{i} \geq S_{\underline{m}_{n}}-\left(\bar{m}_{n}-\underline{m}_{n}\right) \geq \varepsilon_{n} n^{1 /(\tau-1)}-\bar{m}_{n} .
$$

Since $\bar{m}_{n}=a_{n} \varepsilon_{n}^{-\kappa}$,

$$
\begin{aligned}
S_{i} & \geq \varepsilon_{n} n^{1 /(\tau-1)}-a_{n} \varepsilon_{n}^{-\kappa}=\varepsilon_{n} n^{1 /(\tau-1)}-\varepsilon_{n}^{-\kappa} n^{(\tau-2) /(\tau-1)} \\
& \geq \varepsilon_{n} n^{1 /(\tau-1)} / 2,
\end{aligned}
$$

where the last inequality follows because we can take for $\varepsilon_{n}$ some negative power of $\log n$ and since $(\tau-2) /(\tau-1)<1 /(\tau-1)$, for $\tau \in(2,3)$. Therefore,

$$
\mathbb{E}_{n}\left[\mathbb{1}_{\mathcal{E}_{n}} \sum_{i=\underline{m}_{n}+1}^{\bar{m}_{n}} I_{i}\right] \leq\left(\frac{2}{\varepsilon_{n} n^{1 /(\tau-1)}}\right) \sum_{i=\underline{m}_{n}+1}^{\bar{m}_{n}} \mathbb{E}_{n}\left[B_{i}\right] .
$$

For every $i \leq n / 2$,

$$
\mathbb{E}_{n}\left[B_{i}\right] \leq \sum_{v \in[n]} \frac{D_{v}^{2}}{L_{n}(i)},
$$

where, with $\left(D_{(i: n)}\right)_{i \in[n]}$ the order statistics of $\left(D_{j}\right)_{j \in[n]}$ and since $D_{j} \geq 2$ for every $j \in[n]$,

$$
L_{n}(i)=\sum_{j \leq n-i-1} D_{(j: n)} \geq n .
$$

We conclude that, by (2.8) and since $a_{n} n^{-1 /(\tau-1)-1+2 /(\tau-1)}=1$,

$$
\begin{aligned}
\mathbb{E}_{n}\left[\mathbb{1}_{\mathcal{E}_{n}} \sum_{i=\underline{m}_{n}+1}^{\bar{m}_{n}} I_{i}\right] & \leq \bar{m}_{n}\left(\frac{2}{\varepsilon_{n} n^{1 /(\tau-1)}}\right) \frac{1}{n} \sum_{v \in[n]} D_{v}^{2} \\
& =O_{\mathbb{P}}\left(\varepsilon_{n}^{-(1+\kappa)}\right) a_{n} n^{-1 /(\tau-1)-1+2 /(\tau-1)} \\
& =O_{\mathbb{P}}\left(\varepsilon_{n}^{-(1+\kappa)}\right)=o_{\mathbb{P}}(\sqrt{\log n})
\end{aligned}
$$

whenever $\varepsilon_{n} \gg(\log n)^{-1 /(2+2 \kappa)}$. This completes the proof of (2.21), and thus corrects the proof of the required weaker version of [4], Proposition 4.6(a).

Acknowledgment. We thank Justin Salez for pointing out the error to us.

\section{REFERENCES}

[1] Baroni, E., VAn der Hofstad, R. and Komjáthy, J. (2016) Tight fluctuations of weightdistances in random graphs with infinite-variance degrees. Available at http://arxiv.org/ abs/1609.07269.

[2] Baroni, E., VAn der Hofstad, R. and Komjáthy, J. (2017). Nonuniversality of weighted random graphs with infinite variance degree. J. Appl. Probab. 54 146-164. MR3632611 
[3] Bhamidi, S., van der Hofstad, R. and Hooghiemstra, G. (2017). Universality for first passage percolation on sparse random graphs. Ann. Probab. 45 2568-2630.

[4] Bhamidi, S., VAn der Hofstad, R. and Hooghiemstra, G. (2010). First passage percolation on random graphs with finite mean degrees. Ann. Appl. Probab. 20 1907-1965. MR2724425

[5] Feller, W. (1971). An Introduction to Probability Theory and Its Applications. Vol. II. 2nd ed. Wiley, New York. MR0270403

\section{S. BHAMIDI}

DEPARTMENT OF STATISTICS

AND OPERATIONS RESEARCH

304 HANES HALL

UNIVERSITY OF NORTH CAROLINA

Chapel Hill, North CAROLINA 27599

USA

E-MAIL: bhamidi@email.unc.edu
R. VAN DER HOFSTAD

DEPARTMENT OF MATHEMATICS AND COMPUTER SCIENCE

EINDHOVEN UNIVERSITY OF TECHNOLOGY P.O. Box 513

5600 MB EINDHOVEN

THE NETHERLANDS

E-MAIL: rhofstad@win.tue.nl

G. HoOghiEmStra

DIAM

DELFT UNIVERSITY OF TECHNOLOGY

MeKelweg 4

2628 CD DELFT

THE NETHERLANDS

E-MAIL: g.hooghiemstra@tudelft.nl 